\title{
Design of a compliantly actuated exo-skeleton for an impedance controlled gait trainer robot.
}

\author{
Herman van der Kooij, Jan Veneman, Ralf Ekkelenkamp
}

\begin{abstract}
We have designed and built a lower extremity powered exo-skeleton (LOPES) for the training of post-stroke patients. This paper describes the philosophy behind the design of LOPES (Fig. 1), motivates the choices that have been made and gives some exemplary results of the ranges of mechanical impedances that can be achieved.
\end{abstract}

\section{INTRODUCTION}

$\mathrm{I}_{\mathrm{c}}^{\mathrm{n}}$ exoskeletons safe physical interaction with humans is crucial. Actuated exoskeletons are used in robotics that aid neuro rehabilitation of for example stroke patients, paraplegics, and children with cerebral palsy. Robotic aided neuro rehabilitation is an emerging application area in (bio)mechatronics. Traditional physical therapy is labor intensive and physically demanding for the therapists. Robotic technology is a promising aid to relieve the physical therapist, which we possibly really need due to graying of the western population. With robotic aids new possibilities to physically support or constrain patients can be exploited. Also during well defined tasks much more of the patient's actions can be measured and fed back to the patient in innovative therapy programs that are founded in recently gained insights in impaired motor control and motor learning and go far beyond traditional therapy.

\section{GENERAL PHILOSOPY FOR THE DESIGN OF A GAIT TRAINER}

We believe that the design of robots for neurorehabilitation should be based on clinical insights in the principles of successful training methods, which can be derived from clinical practice and from the biomechanical and control basics of human walking. The clearest determinant of success of gait training in stroke patients appeared to be the amount of training: earlier gait training yields better and faster recovery [2]. Three further aspects of the training appear to be important: task specificity, active patient participation, and selectivity of support

\section{A. Task Specificity}

There is increasing evidence that 'task specific' training leads to better results in the relearning of motor functions [3]. A training is 'task specific' when the functions that have

This study was supported by a Vedi grant (016027011) of the Netherlands Organisation of Scientific research and by the institute for biomedical technology of the University of Twente.

The authors are at the University of Twente, PO Box 217, $7500 \mathrm{AE}$, NL (phone: +31-53-4894779; fax:+31-53-4893695; e-mail:

h.vanderkooij@utwente.nl) to be (re-) learned are actually practiced in training, as naturally as possible. This is in contrast to for example strength or endurance training, although these can have additional value. This starting-point is likely to apply also for the rehabilitation of walking in stroke patients [4-7]. For gait training it is then of critical importance to walk repetitively in a 'natural' manner and with the correct proprio- and extero-ceptive information [8-10]. This implies that the best training is simply to walk around as much as possible. However, heavily impaired stroke patients are not able to walk safely, and it is also difficult for a therapist to make leg- or posture-corrections during overground walking. In these cases additional devices might be helpful. For training with such a device it still applies that walking should resemble free walking as close as possible.

\section{B. Active patient participation}

For an effective task specific training, active participation of the patient in task realization is essential; passive training regimes where motions are imposed without effort of the patient appear to have less effect $[11,12]$. It is an open question how active participation can optimally be enforced by a device. Therapists use for example strategies like demonstrating a motion or by enforcing it, and then motivating the patient to do it unassisted, reducing their manual assistance. It has also been shown that transient error augmentation can lead to faster motor control learning [13], which suggests related rehabilitation strategies.

\section{Selectivity}

Gait has to be considered as a complex and dynamical control problem [14], realizing several goals simultaneously, like: keep the body upright, drive the body forward, reduce

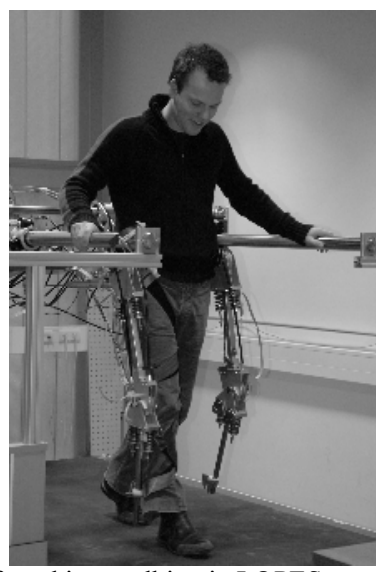

Fig. 1: Picture of a subject walking in LOPES 
impacts, keep lateral stability, and so on [15]. Such subtasks can possibly be trained separately. Task specific training can therefore also mean training on a selective number of these subtasks, while initially omitting others, for learningtransparency or safety reasons. 'Transparency' means that the patient is helped in focusing on a specific subtask, a process then facilitated by the robot taking care of other subtasks. Walking is anything but a simple motion profile that has to be learned.

Besides this training of subtasks selectivity can also be implemented as training or assistance of selected joints. In stroke patients it usually occurs that one leg is functioning quite well, while the other is heavily impaired. Also other options can be kept in mind, like error augmentation on one specific joint, to make a patient aware of his motion-'errors'.

Beside this general philosophy there is little evidence for the superiority of specific training methods. A training robot should therefore ideally also offer possibility to implement new training strategies, to perform clinical research on particular training effects and be flexible for adaptation to the individual patient's needs or the individual therapist's insights.

A gait training device always implies changes compared to free walking, so the real question is, with what device and with what kind of training most transfer of ability to the desired tasks, i.e. over ground walking, takes place. This can only be determined by doing clinical research inside an existing device.

\section{Existing gait trainer robots}

A few gait training devices have already been developed that fit in the presented outline. The most important commercially available devices, are the Lokomat, the Gait Trainer [16] and the AutoAmbulator. About the last regrettably little to nothing has been published. A limitation of the Lokomat is that it is basically position controlled, so that it is only poorly possible to give slight or no support at all in order to let the patient walk freely. This limits the possibilities for active participation of the patient. Recently implementation of control schemes that facilitate 'human cooperative strategies' or impedance control were presented [17], but it is not yet described clearly how well the relatively heavy and high friction Lokomat-device performs in this field. Furthermore, only sagittal motions of the legs are allowed, whereas the pelvis/trunk of the patient is fixated. This gives the possibility to enforce gait-like motions upon the legs in the sagittal plane, but the dynamic gait task is severely altered [18].

A limitation of the Gait Trainer is that the only interaction takes place at the foot sole so that typical poor joint stability of stroke patients cannot be controlled, for example to prevent hyperextension of the knee. Furthermore the cutaneous input at the foot sole with such a system is unnatural, which might disturb training effectivity. Also it is not possible to deviate from the prescribed trajectory in space, only in timing, although current developments take the direction of fully 'programmable' (haptic) footplates
[19].

Another interesting approach is the use of robotic orthoses during body weight supported treadmill training (BWSTT) as motivated in [20]. As advantage over the aforementioned devices the possible active assistance to the ankle joint is stressed. It is claimed that using active ankle assistance might cause normalized gait characteristics of spinal cord injury (SCI) patients..

\section{E. Possible improvements on existing gait trainer robots}

For the aforementioned commercial devices there are experimental indications that stroke patients benefit by their use, although no profound clinical evaluation has taken place yet [21]. We believe that improvements are possible on all three stated training aspects - task specificity, active participation and selectivity, compared to existing devices. This can be realized by creating a robot that is basically 'invisible' in the sense that it is not hindering the patient in any way, so that the person uses it experiences to walk actively and freely. Upon this basis, selected supportive actions should then be added. This could be realized by a robot that resembles a haptic device, in the sense that its mechanical behavior ('impedance') has to be adjustable. This would allow implementing several training regimes. For example either resistance or assistance could be offered to the patient. It also would allow using the robot for gait observation and measurements [22]. This way the robot imitates the role of the therapist during BWSTT.

Another improvement would be adding degrees of freedom, so that certain subtasks of walking will not be omitted, and the natural dynamics of walking can be exploited and patients can get accustomed to these dynamics again.

\section{FunCtional DESCRIPTION OF LOPES}

A principle choice we made was to build an exoskeleton. An advantage of an exoskeleton is that it moves in parallel to the skeleton of the patient, so that no additional degrees of freedom are needed to follow patient motions. If for example the lower leg should be guided by a robot arm from the fixed world, comparable to what a therapist does, this arm would need the full six degrees of freedom to follow the leg motion in global space. Moreover, the lower leg has a large range of motion in global space. This would apply for each limb. An exoskeleton only needs approximately the same ranges of motion as the patients' joints. The fact that the exoskeletonjoint-motions directly correspond with the motions of the patients' joints makes it also easier to implement mechanical safety limits to motion- and torque-ranges. Use of an exoskeleton does introduce the need to align the axes of patient and exoskeleton joints.

Different from common orthoses, LOPES's exoskeleton is connected to the fixed world, at pelvis height. This makes it possible to compensate for the weight of LOPES, and to apply external corrective joint torques or forces to the patient's pelvis. This yields more and simpler possibilities to 
prevent a patient from falling.

As said, the exoskeleton needs all degrees of freedom that are essential for walking. Introducing all degrees of freedom of the human body would make the construction unnecessarily complex. Not every possible leg and pelvis motion of a human is needed for walking. It is however difficult to decide which DoF can be omitted. Experiments we did with walking on a treadmill while the pelvis was fixed, as is the case in some robotic rehabilitation settings, pointed out that this fixation changes walking probably too much compared to free walking to offer a proper taskspecific training. Rotations of the pelvis during walking are small, so those can probably be realized by the inevitable play in the connections. Based on experiments with walking inside a first experimental prototype we also concluded that the abduction of the legs, that is the hip rotation making the leg move sideways, cannot be omitted for proper walking. Fig. 2 shows the DoFs of LOPES that will be constrained, unconstrained or actuated by powered actuators.

\section{ACtUATION OF LOPES}

As aforementioned the robot should be suited for interaction control. The choice of basic control outline for interaction control has influence on optimal design choices. In general there are two ways to realize an interactive/haptic/kinaesthetic display [23]; impedancecontrol-based, that is 'measure position and display force' and admittance-control-based, that is 'measure force and display position', although hybrid forms exist

The important difference is that in impedance-control the quality of the display will depend on the accuracy of the position measurement and the bandwidth and accuracy of the force servos, and in case of admittance-control on the accuracy of the force measurement and the bandwidth and accuracy of the position servo. The choice is basically about a lightweight (and flexible) uncompensated construction (device dynamics outside the feedback control loop) versus a rigid (and heavy) controller-compensated construction

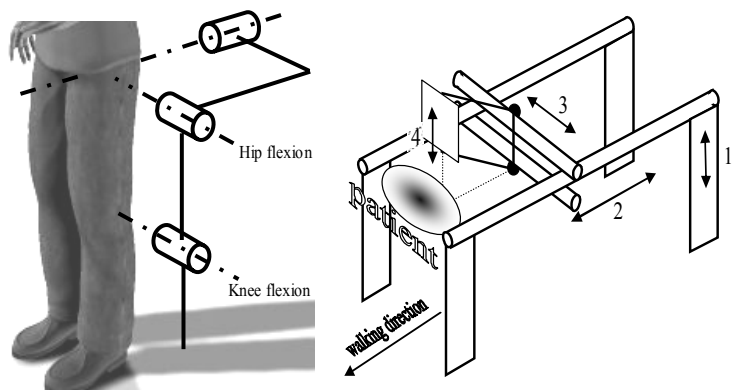

Fig. 2: Degrees of Freedom of LOPES. Left: DoFs of the exoskeleton that are actuated; other DoFs are left free (ankle, knee ab/aduction) or constrained (hip and knee endo/exorotation). Right: schematic drawing of the construction for connecting the exo-skeleton to the fixed world consisting of height adjustable frame (1), two sets of parallel bars with carriages for the for-/backward (2) and sideways (3) motion that are both actuated, and a parallelogram with weight compensation that allows for vertical pelvic motion as occurs during walking. (device dynamics inside the feedback control loop).

For our robot, impedance control has been chosen as basic control outline. The main reason for this choice was the advantage concerning patient and therapist safety. An impedance display needs less actuator power and force as no high power/precision inner position loop is needed. Also the robot construction can be more compliant and thus lighter, again because a rigid position control is not needed. Impedance control furthermore easier allows stable 'zero' impedance control. Finally, impedance control makes the programmed dynamical behavior available everywhere on the construction (at least in every actuated degree of freedom), while in admittance control impedance is only available at the force sensors - usually the end effector. For the robot design the choice for impedance control implies that the moving parts of the construction should be lightweight and the actuators 'pure' force sources.

These both demands were combined in the actuator design (Fig. 3) for the robot [1]. The basic idea of this design was to remove the actual motors from the moving exoskeleton to reduce weight, or to keep weight low, while applying motors with more power. The power is then transferred from motor to joint by use of flexible Bowden-cable transmissions, to allow free motion of the joints. Finally, at the joints a compliant coupling, e.g. a set of springs, is used to create a proper force source at these joints, using an inner force feedback loop, following the principles of Series Elastic Actuation [24].

\section{MECHANICAL IMPEDANCE IN PATIENT AND THERAPIST IN CHARGE MODE}

In the patient in charge mode the subject attached to LOPES has to walk unhindered. In this mode LOPES' mechanical impedance should be as low as possible. As a typical example the torque of a joint controlled to zero force while moving the joint is displayed in Fig. 4. The friction in the Bowden cables can not be compensated fully by the controller. The remaining friction is so low that it was not perceived by subjects walking in LOPES. Our first subjective impression was that subjects walking in LOPES in the low impedance mode walked natural. The subjects reported that walking in LOPES was comfortable and did not hinder their motion. With EMG and full kinematical motion analysis we will quantify how walking is affected in

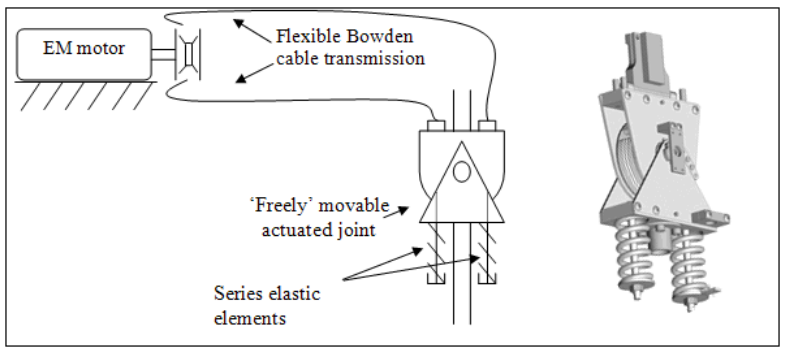

Fig. 3: Schematic and solid working drawings of Bowden cables driven series elastic joint actuator that actuates the exoskeleton. 
the patient in charge mode.

In the therapist in charge mode it is important that LOPES can generate sufficient stiffness to stabilize the walking pattern. To quantify the impedance of LOPES the mechanical end point impedance of the leg in the sagittal plane has been estimated. For this purpose one side of the hip joint was attached to the fixed world whereas the lower part of LOPES 'shank' was attached to a robotic device that provided a pseudo-random position perturbation to LOPES's 'shank', whereas LOPES was programmed to maintain a constant stiffness in Cartesian global space. With Virtual Model Control LOPES can generate a stiffness of $5 \mathrm{kN} / \mathrm{m}$ at low frequencies (Fig. 5). Translating this stiffness to a vertical force acting on the pelvis will give an upward force of $500 \mathrm{~N}$ for each $\mathrm{cm}$ of downward motion of the pelvis. The achieved stiffness is thus sufficient to compensate for body weight. In Fig. 5 the effect of the mass can be recognized from 1-2 Hz. The lower the reference stiffness was, the higher the frequency we achieved upon which LOPES has an ideal stiffness. Preliminary experiments showed that LOPES in combination with VMC can selectively modulate aspects of gait such as foot clearance and step length [25].

\section{CONCLUSIONS}

With LOPES we designed and built a light weight impedance controlled exoskeleton for gait therapy. LOPES creates a safe environment in which the patient is free to explore his capabilities and learn new skills without falling.

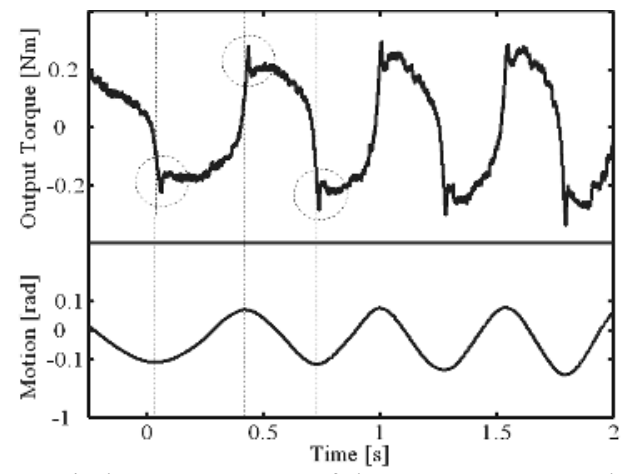

Fig. 4: Typical torque response of the actuator to external motion, while controlled to zero force output. The small peaks are caused by stick, appearing when the motion is reversed (from [1]).
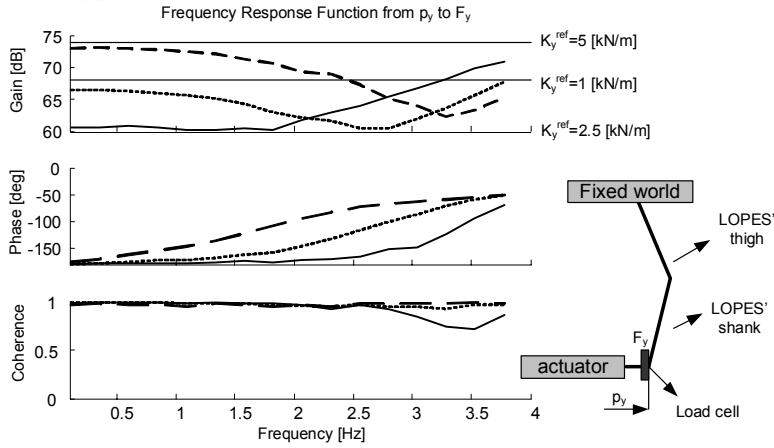

Fig. 5: Typical torque response of the actuator to external motion, while controlled to a desired stiffness $\left(\mathrm{K}_{\mathrm{y}}{ }^{\text {ref }}\right)$.
Compared to other exoskeletons developed for gait graining LOPES has more actuated degrees of freedom, supports a larger range of impedances so that both unhindered walking (patient in charge mode) as fully passive walking (robot in charge mode) is possible.

\section{REFERENCES}

[1] J. F. Veneman, R. Ekkelenkamp, R. Kruidhof, F. C. T. van der Helm, and $\mathrm{H}$. van der Kooij, "A series elastic- and Bowden-cable-based actuation system for use as torque actuator in exoskeleton-type robots," International Journal of Robotics Research, vol. 25, pp. 261$281,2006$.

[2] G. Kwakkel, R. van Peppen, R. C. Wagenaar, S. W. Dauphinee, C Richards, A. Ashburn, K. Miller, N. Lincoln, C. Partridge, I. Wellwood, and P. Langhorne, "Effects of augmented exercise therapy time after stroke - A meta-analysis," Stroke, vol. 35, pp. 2529-2536, 2004.

[3] N. A. Bayona, J. Bitensky, K. Salter, and R. Teasell, "The role of taskspecific training in rehabilitation therapies," Top Stroke Rehabil, vol. 12, pp. 58-65, 2005

[4] C. L. Richards, F. Malouin, and C. Dean, "Gait in stroke - Assessment and rehabilitation," Clinics in Geriatric Medicine, vol. 15, pp. 833-+, 1999.

[5] F. Malouin, C. L. Richards, B. McFadyen, and J. Doyon, "To walk again after a stroke: new perspectives of locomotor rehabilitation," M S-Medecine Sciences, vol. 19, pp. 994-998, 2003.

[6] S. H. Peurala, I. M. Tarkka, K. Pitkanen, and J. Sivenius, "The effectiveness of body weight-supported gait training and floor walking in patients with chronic stroke," Arch Phys Med Rehabil, vol. 86, pp. 1557-64, 2005.

[7] R. F. Macko, F. M. Ivey, L. W. Forrester, D. Hanley, J. D. Sorkin, L. I. Katzel, K. H. Silver, and A. P. Goldberg, "Treadmill Exercise Rehabilitation Improves Ambulatory Function and Cardiovascular Fitness in Patients With Chronic Stroke. A Randomized, Controlled Trial," Stroke, 2005.

[8] B. H. Dobkin, "Strategies for stroke rehabilitation," Lancet Neurol, vol. 3, pp. 528-36, 2004.

[9] H. Barbeau, "Locomotor training in neurorehabilitation: emerging rehabilitation concepts," Neurorehabil Neural Repair, vol. 17, pp. 311, 2003.

[10] V. Dietz and J. Duysens, "Significance of load receptor input during locomotion: a review," Gait Posture, vol. 11, pp. 102-10, 2000.

[11] M. Lotze, C. Braun, N. Birbaumer, S. Anders, and L. G. Cohen, "Motor learning elicited by voluntary drive," Brain, vol. 126, pp. 866872, 2003.

[12] A. Kaelin-Lang, L. Sawaki, and L. G. Cohen, "Role of voluntary drive in encoding an elementary motor memory," J Neurophysiol, vol. 93 , pp. 1099-103, 2005.

[13] J. L. Emken and D. J. Reinkensmeyer, "Robot-enhanced motor learning: Accelerating internal model formation during locomotion by transient dynamic amplification," Ieee Transactions on Neural Systems and Rehabilitation Engineering, vol. 13, pp. 33-39, 2005.

[14] H. van der Kooij, R. Jacobs, B. Koopman, and F. van der Helm, "An alternative approach to synthesizing bipedal walking," Biol Cybern, vol. 88, pp. 46-59, 2003.

[15] J. Perry and B. Schoneberger, Gait analysis: normal and pathological function: Thorofare, NJ: SLACK Incorporated, 1992.

[16] J. Hidler, D. Nichols, M. Pelliccio, and K. Brady, "Advances in the understanding and treatment of stroke impairment using robotic devices," Top Stroke Rehabil, vol. 12, pp. 22-35, 2005.

[17] R. Riener, L. Lunenburger, S. Jezernik, M. Anderschitz, G. Colombo, and V. Dietz, "Patient-cooperative strategies for robot-aided treadmill training: First experimental results," Ieee Transactions on Neural Systems and Rehabilitation Engineering, vol. 13, pp. 380-394, 2005.

[18] J. M. Hidler and A. E. Wall, "Alterations in muscle activation patterns during robotic-assisted walking," Clin Biomech (Bristol, Avon), vol. 20, pp. 184-93, 2005.

[19] H. Schmidt, D. Sorowka, S. Hesse, and R. Bernhardt, "[Development of a robotic walking simulator for gait rehabilitation]," Biomed Tech (Berl), vol. 48, pp. 281-6, 2003. 
[20] D. P. Ferris, G. S. Sawicki, and A. R. Domingo, "Powered lower limb orthoses for gait rehabilitation," Topics in Spinal Cord Injury Rehabilitation, vol. 11, pp. 34-49, 2005.

[21] C. Werner, S. Von Frankenberg, T. Treig, M. Konrad, and S. Hesse, "Treadmill training with partial body weight support and an electromechanical gait trainer for restoration of gait in subacute stroke patients: a randomized crossover study," Stroke, vol. 33, pp. 2895$901,2002$.

[22] J. M. Hidler and S. Goldberg, " Inverse-dynamics based assessment of walking using a robotic gait orthosis," Gait \& Posture, vol. 21, pp. S131, 2005.

[23] R. J. Adams and B. Hannaford, "Control law design for haptic interfaces to virtual reality," IEEE Transactions on Control Systems Technology, vol. 10, pp. 3-13, 2002.

[24] J. Pratt, B. Krupp, and C. Morse, "Series elastic actuators for high fidelity force control," Industrial Robot, vol. 29, pp. 234-241, 2002.

[25] R. Ekkelenkamp, J. Veneman, and H. van der Kooij, "LOPES: selective control of gait functions during the gait rehabilitation of CVA patients," presented at Rehabilitation Robotics, 2005. ICORR 2005. 9th International Conference on, 2005. 